\title{
Pipeline Diameter Significantly Impacts the Long-Term Fate of Jailed Side Branches during Treatment of Intracranial Aneurysms
}

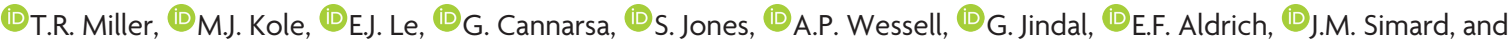

(1DD. Gandhi

\begin{abstract}
BACKGROUND AND PURPOSE: Although covered side branches typically remain patent acutely following Pipeline Embolization Device embolization of intracranial aneurysms, the long-term fate of these vessels remains uncertain. We therefore elected to investigate factors that may influence the long-term patency of these covered side branches.
\end{abstract}

MATERIALS AND METHODS: We retrospectively evaluated the long-term patency of side branches covered by the Pipeline Embolization Device at our institution during treatment of intracranial aneurysms with at least 6 months of conventional angiography follow-up. Procedural and anatomic factors that might influence the fate of covered side branches were explored.

RESULTS: One hundred forty-eight Pipeline Embolization Device treatments in 137 patients met the inclusion criteria. In 217 covered side branches, 29 (13.4\%) were occluded on follow-up, and 40 (18.4\%) were stenotic. All stenoses and occlusions were asymptomatic. In the entire cohort and in the largest subset of ophthalmic arteries, a smaller Pipeline Embolization Device diameter was associated with branch vessel occlusion $(P=.001, P=.013)$. When we considered stenotic and occluded side branches together, smaller Pipeline Embolization Device size $(P=.029)$ and administration of intraprocedural abciximab $(P=.03)$ predicted side branch stenosis/occlusion, while anterior choroidal branch type $(P=.003)$ was a predictor of gross side branch patency.

CONCLUSIONS: A smaller Pipeline Embolization Device diameter is associated with delayed side branch stenosis/occlusion following Pipeline Embolization Device treatment, likely due to the higher metal density of smaller caliber devices. Although hemodynamic factors, including the potential for collateral flow, are still paramount in determining the fate of covered side branches, the amount of metal coverage at the side branch orifice also plays an important role.

ABBREVIATION: PED = Pipeline Embolization Device

$F$ ow diversion using the Pipeline Embolization Device (PED; Covidien, Irvine, California) is an effective treatment for intracranial aneurysms. ${ }^{1-5}$ Although originally indicated for large/ giant wide-neck aneurysms arising from the internal carotid artery proximal to the posterior communicating artery origin, flow diversion is increasingly used for the treatment of smaller aneurysms and those arising from more distal vessels and the posterior circulation. ${ }^{6-9}$ The PED was designed to allow progressive thrombosis and remodeling of cerebral aneurysms while preserving flow in arterial side branches covered by the device. Although most covered side branches have been shown to remain patent acutely

Received April 27, 2018; accepted after revision August 28.

From the Departments of Diagnostic Radiology (T.R.M., G.J., D.G.), Neuroradiology (G.J., D.G.) and Neurosurgery (M.J.K., E.J.L., G.C., S.J., A.P.W., G.J., E.F.A., J.M.S., D.G.), University of Maryland Medical Center, Baltimore, Maryland.

Please address correspondence to Timothy R. Miller, MD, University of Maryland Medical Center, Department of Diagnostic Radiology, Room N2W78, 22 South Greene St, Baltimore, Maryland 21201; e-mail: Tmiller5@umm.edu

http://dx.doi.org/10.3174/ajnr.A5863 following PED deployment, the long-term fate of these vessels remains uncertain. Prior reports have noted varying rates of delayed branch vessel occlusion following treatment with the PED, and the factors that determine long-term patency are unclear. ${ }^{10-14}$

We therefore elected to retrospectively evaluate the long-term patency of arterial side branches covered by the PED at our institution during the treatment of intracranial aneurysms. Procedural and anatomic factors that may influence the fate of covered side branches were explored.

\section{MATERIALS AND METHODS}

The protocol of this retrospective study was approved by the University of Maryland, Baltimore, institutional review board (HP-00077364). From a prospectively maintained clinical data base, we retrospectively identified all intracranial aneurysm treatments using the PED at our institution in which there was device coverage of $\geq 1$ arterial side branch and the patient had undergone at least 1 follow-up catheter angiogram $\geq 6$ months follow- 
ing flow-diverter placement. Information in the clinical data base was obtained from multiple sources. Patient demographics, clinical outcomes, and procedural details were acquired from procedure reports and the electronic medical record. Aneurysm and parent vessel characteristics and imaging follow-up of lesions, covered side branches, and parent vessels were obtained by review of the relevant catheter angiograms as detailed below.

Factors specifically evaluated for impact on branch vessel patency rates included the following: patient age, sex, rupture status, prior aneurysm treatment, procedure time, fluoroscopy time, number of PEDs deployed, use of a J-tip microwire or balloon angioplasty to achieve adequate device apposition, the use of the first-versus-second generation PED, maximum aneurysm sac size, aneurysm neck size, minimum and maximum parent vessel diameter, differences in the size of PEDs deployed, the minimum and maximum parent vessel diameters, branch vessel incorporation into the aneurysm sac, degree of periprocedural platelet inhibition as measured by the VerifyNow P2Y12 assay (Accumetrics, San Diego, California), intraprocedural administration of abciximab for intraprocedural platelet aggregation, the development of endothelial hyperplasia resulting in the PED construct on follow-up, length of DSA follow-up, and type of covered branch vessel. Patient-specific variables, such as smoking status or history of hypertension, were not included in the analysis because these data points were not collected in the prospective data base. Variables obtained by review of follow-up imaging, such as branch vessel stenosis/occlusion or the presence of endothelial hyperplasia, were evaluated only on the most recent catheter angiograms.

All relevant catheter angiograms were reviewed by at least 2 team members (T.R.M., M.J.K., E.J.L., S.J., G.C., G.J., D.G.), with any discrepancies in measurements obtained adjudicated by 2 attending neurointerventionalists (T.R.M., D.G.) with 6 and 13 years of experience. Aneurysm sac and neck sizes were measured on relevant $2 \mathrm{D}$ and $3 \mathrm{D}$ rotational catheter angiograms. In most instances, minimal and maximal parent vessel diameters were obtained from measurements made during PED treatment on magnified 2D angiography "working angle" views of the PED landing site and sent to the PACS. Multiple measurements were typically acquired by the treating interventionalist to ensure appropriate device sizing, including at the proximal and distal device landing sites. These measurements were often verified by inspection of the source imaging of $3 \mathrm{D}$ rotational angiography, which provides a cross-sectional view of the parent vessel. In instances in which intraprocedural measurements were not stored on the PACS, they were acquired by at least 2 members independently using all relevant imaging sources (at a minimum, the magnified 2D working angle angiography views and 3D rotational angiography source images). Then, the adequacy of device apposition was determined intraprocedurally by the treating neurointerventionalist by review of $2 \mathrm{D}$ rotational angiography imaging performed after device deployment. Occasionally, flat panel CT was performed to confirm the adequacy of device apposition. Finally, the degree of intimal hyperplasia on follow-up imaging was determined by measuring the gap between the PED and the patent vessel lumen on magnified 2D conventional angiography (best appreciated on nonsubtracted, native views).

\section{Treatment Protocol}

Treatment was performed by 1 of 3 neurointerventional radiologists (D.G., G.J., T.R.M.) at a single medical center using general endotracheal anesthesia. A dual antiplatelet regimen of aspirin and clopidogrel was initiated 2-4 weeks before treatment, with subsequent titration of clopidogrel dosing based on platelet inhibition testing using the P2Y12 assay (target range, 60-200 P2Y12 reaction units). Access was obtained using a triaxial system, beginning with placement of a $6 \mathrm{~F}-7 \mathrm{~F}$ shuttle sheath into the target cervical vessel. This was followed by navigation of an intermediate guide catheter, such as a 058 Navien distal intracranial support catheter (Covidien), through the shuttle to the skull base. Finally, through the intermediate catheter, PED deployment was performed using an 0.027 microcatheter such as the Marksman or Phenom (Covidien).

Placement of 1 PED was generally considered sufficient for aneurysm treatment in most cases, with deployment of multiple devices reserved primarily for instances of incomplete lesion coverage with the first device. Adjunctive coiling was used in cases in which the target lesion demonstrated high-risk features (eg, recent growth, irregular morphology) or if the risk of delayed rupture was thought to be significant (lesion size, $\geq 13 \mathrm{~mm}$ ). Inadequate apposition of the PED with the parent vessel wall was treated with either a J-tip microwire and/or balloon angioplasty using a compliant balloon. Delayed angiography of the parent vessel was performed 10-15 minutes following PED placement to evaluate platelet aggregation, which, if present, was treated with abciximab. Postprocedure, patients were closely monitored overnight in an intensive care level unit and discharged after 1-2 days of postoperative observation.

\section{Follow-Up Protocol}

All patients were evaluated in our neurointerventional clinic 1-3 weeks following treatment. Dual antiplatelet therapy was continued for at least 6 months following the procedure, with continued adjustment of clopidogrel dosing based on the results of platelet inhibition testing (again, with a goal of 60-200 P2Y12 reaction units). Initial imaging follow-up of the treatment site was typically performed at 3 months using either MR imaging or CT angiography. If there were no clinical or imaging concerns, follow-up conventional angiography was then performed 6-12 months postPED deployment. More long-term follow-up of the treatment site varied on the basis of the status of the target aneurysm and parent vessel, as well as the presence of additional intracranial aneurysms. However, most patients underwent additional follow-up imaging at 18-36 months.

\section{Statistical Analysis}

Data are presented as mean and range for continuous variables and frequency for categoric variables. Univariate analysis was performed using unpaired $t$ tests and $\chi^{2}$ tests as appropriate. Factors predictive in univariate analysis $(P \leq .1)$ were then evaluated by multivariate regression analysis. To account for repeat measurements, we used a generalized estimating equation, centered on patients, with a logistic regression model and an exchangeable correlation matrix. $P$ values $<.05$ were considered statistically 
Table 1: Cohort demographics

\begin{tabular}{lc}
\hline \multicolumn{2}{c}{ Demographics } \\
\hline No. of patients & 137 \\
No. of PED treatments & 148 \\
Average patient age (yr) & 54.7 \\
\% Male per treatment & $6.6 \%$ \\
No. of aneurysms treated & 178 \\
Average aneurysm size (mm) & 5.2 \\
\% Aneurysms treated acutely/subacutely after rupture & $3.3 \%$ \\
\% Aneurysms with branch vessel incorporation & $35 \%$ \\
Mean No. of PEDs per treatment & 1.07 \\
\% Aneurysms adjunctively coiled & $9.2 \%$ \\
\% Treatments using J-tip microcatheter & $17 \%$ \\
\% Treatments using balloon angioplasty & $6.1 \%$ \\
\% Treatments requiring abciximab & $4.8 \%$ \\
Mean length of DSA follow-up (mo) & 18.4 \\
\hline
\end{tabular}

significant. Statistical analysis was performed using STATA, Version 12.0 (StataCorp, College Station, Texas).

Receiver operating characteristic analysis was performed for continuous variables significantly associated with branch vessel occlusion in multivariate analysis. The receiver operating characteristic curves were plotted, and area under the curve values for relevant parameters were calculated and compared. Thresholds for optimal sensitivity and specificity were also calculated for relevant parameters.

\section{RESULTS}

Two hundred forty-two intracranial aneurysm treatments in 196 patients were performed at our institution using the PED from November 2011 to the time of data acquisition in October 2017. Of these, 148 PED treatments in 137 patients met the inclusion criteria. Twenty-nine cases were excluded due to a lack of covered side branches, while 65 were excluded due to insufficient follow-up (Table 1). Ten patients underwent 2 PED treatments of aneurysms located at different sites, while 3 patients underwent placement of a second PED 2-3 years after their initial treatment due to persistence of the target aneurysm. In these instances, follow-up of the first PED was considered to end at the time of placement of the second device.

A total of 178 intracranial aneurysms were treated in the cohort, with an average maximum sac diameter of $5.2 \mathrm{~mm}$ (range, $1-21 \mathrm{~mm}$ ) and average neck width of $3.3 \mathrm{~mm}$ (range, $1-11 \mathrm{~mm}$ ). Thirty-nine aneurysms had been previously treated by coil embolization (22\%), while 3 had undergone prior microsurgical clipping. Five aneurysms in the cohort were treated in the acute-tosubacute period range: few days to one week following rupture because they were not amenable to more traditional treatment methods such as coil embolization or microsurgical repair. Finally, a branch artery was incorporated into the neck or body of 61 treated aneurysms (34\%).

Most patients were treated with a single PED (average, 1.07 PEDs per treatment), with only 8 cases using 2 devices and a single case using 3 PEDs. Aneurysm neck coverage was complete in all cases except 1 in which a PED migrated into the distal M1 segment of the middle cerebral artery ipsilateral to the target paraclinoid internal carotid artery aneurysms. The patient subsequently underwent successful placement of a second PED for the paraclinoid lesions at a later date. Sixteen aneurysms were adjunctively coiled
Table 2: Branch vessel outcomes by type

\begin{tabular}{lcccc}
\hline \multicolumn{1}{c}{ Type } & No. & Patent & Stenotic & Occluded \\
\hline Ophthalmic artery & 125 & $72 \%$ & $22 \%$ & $5.8 \%$ \\
PcomA & 39 & $56.7 \%$ & $10 \%$ & $33.3 \%$ \\
Anterior choroidal artery & 31 & $96.8 \%$ & $0 \%$ & $3.2 \%$ \\
A1 arterial segment & 11 & $28 \%$ & $36 \%$ & $36 \%$ \\
PICA & 3 & $33.4 \%$ & $66.6 \%$ & $0 \%$ \\
AcomA & 3 & $33.4 \%$ & $0 \%$ & $66.6 \%$ \\
Other $^{\text {a }}$ & 5 & $80 \%$ & $0 \%$ & $20 \%$ \\
\hline
\end{tabular}

Note:-PcomA indicates posterior communicating artery; AcomA, anterior communicating artery.

${ }^{a}$ Detailed in Results section.

at the time of PED placement (9\% of treated lesions in the cohort). In 25 instances, a J-tip microwire was used to improve apposition of the device with the parent vessel wall (17\% of PED treatments), while a balloon catheter was necessary in 9 cases (6.1\%). Finally, abciximab was administered in 7 cases $(4.7 \%$ of treatments) due to signs of early platelet aggregation.

The mean length of conventional angiography follow-up in the cohort was 18.4 months (range, 6-61 months). However, the total length of imaging and clinical follow-up for most patients was significantly longer when including MR imaging and CT angiography of the treatment site. One hundred thirty-two aneurysms (74.2\%) were completely occluded at the last imaging follow-up (DSA, CTA, or MRA), while 36 lesions were incompletely occluded.

Complications in the cohort included 4 instances of periprocedural ischemic events with resulting changes in contralateral strength and/or sensory findings that subsequently resolved, 1 small occipital intraparenchymal hematoma following treatment of a paraophthalmic aneurysm that resulted in a permanent quadranopsia, 2 retroperitoneal hematomas, and 1 instance of catheter-induced dissection of a cervical internal carotid artery during treatment of a paraclinoid aneurysm that healed without consequence. Delayed complications included 1 patient who developed a transient small area of edema and petechial hemorrhage without associated symptoms in the ipsilateral parieto-occipital lobes 2 months following PED treatment of a right paraophthalmic aneurysm. The abnormality resolved on follow-up without treatment. Finally, 1 patient treated for a giant posterior communicating artery aneurysm that recurred after coiling developed worsening contralateral arm and leg weakness due to mass effect after the lesion failed to close with the PED. The patient eventually died from the associated mass effect and edema 21 months after PED treatment. The overall rate of permanent neurologic morbidity and mortality in the cohort was $1.5 \%$ and $0.7 \%$.

In total, there were 217 covered side branches in the cohort (Table 2), most of which were in the anterior circulation (98.2\%). Covered side branches included 125 ophthalmic arteries, 39 posterior communicating arteries, 31 anterior choroidal arteries, 11 A1 anterior cerebral artery segments, 3 posterior inferior cerebellar arteries, 3 anterior communicating arteries, 1 M2 middle cerebral artery branch, 1 P3 posterior cerebral artery branch, 1 lenticulostriate perforator, and 2 anterior temporal arteries arising from the M1 middle cerebral artery segment (Table 2). Of the covered 39 posterior communicating arteries, 8 were of a fetal configuration.

Of 217 covered side branches, 29 (13.4\%) were occluded on 


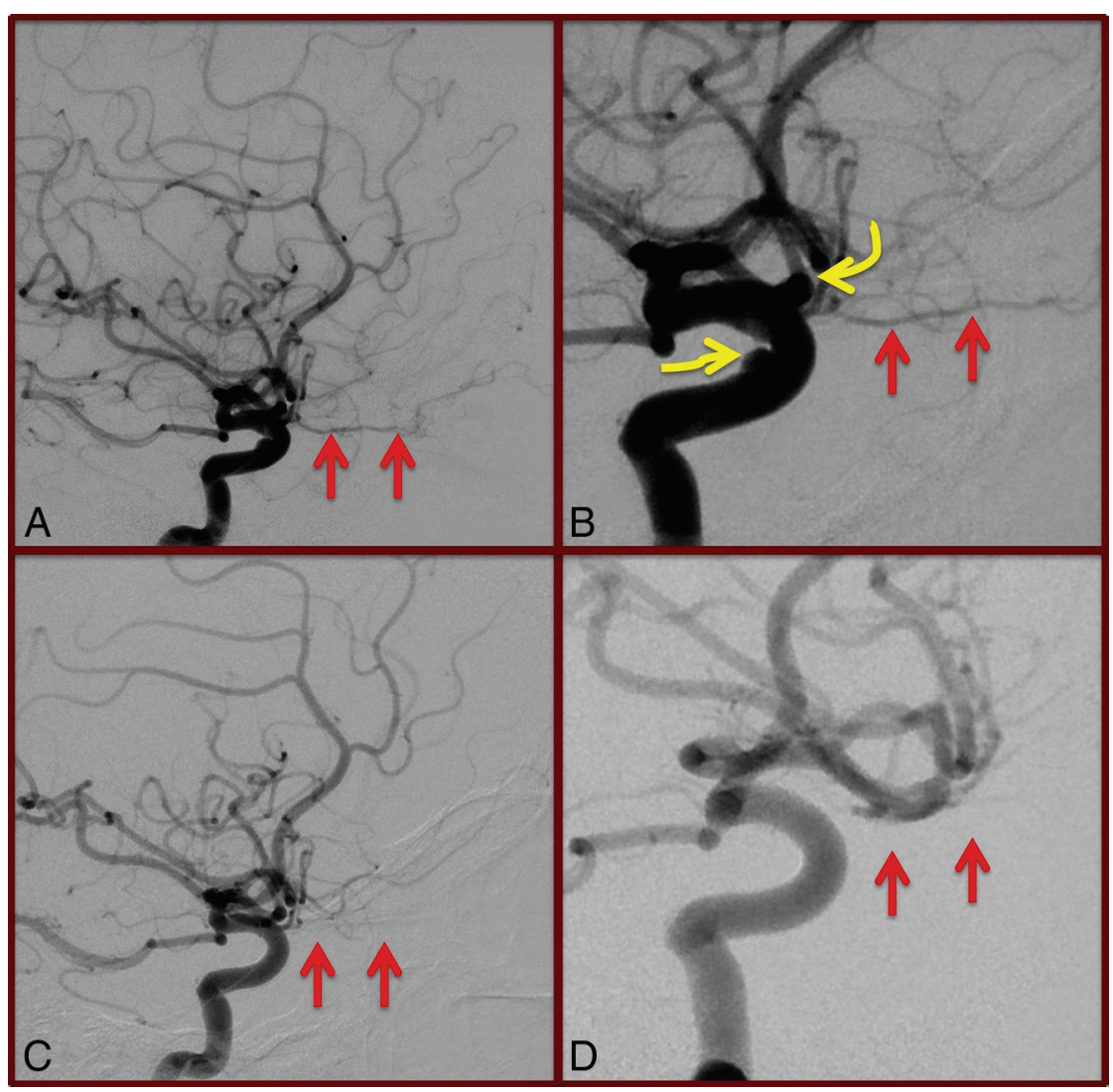

FIG 1. Global and magnified lateral views $(A$ and $B)$ of pre-PED right internal carotid artery angiography demonstrating gross patency of the right ophthalmic artery (straight arrows). The target paraophthalmic and cavernous aneurysms (curved arrows, $B$ ) are also visualized. Conventional angiography performed 6 months following PED deployment $(C$ and $D)$ demonstrates occlusion of the target aneurysms; however, the ophthalmic artery is also occluded (straight arrows). The patient was asymptomatic, and the ophthalmic artery was found to fill retrogradely on external carotid artery injections (not shown).

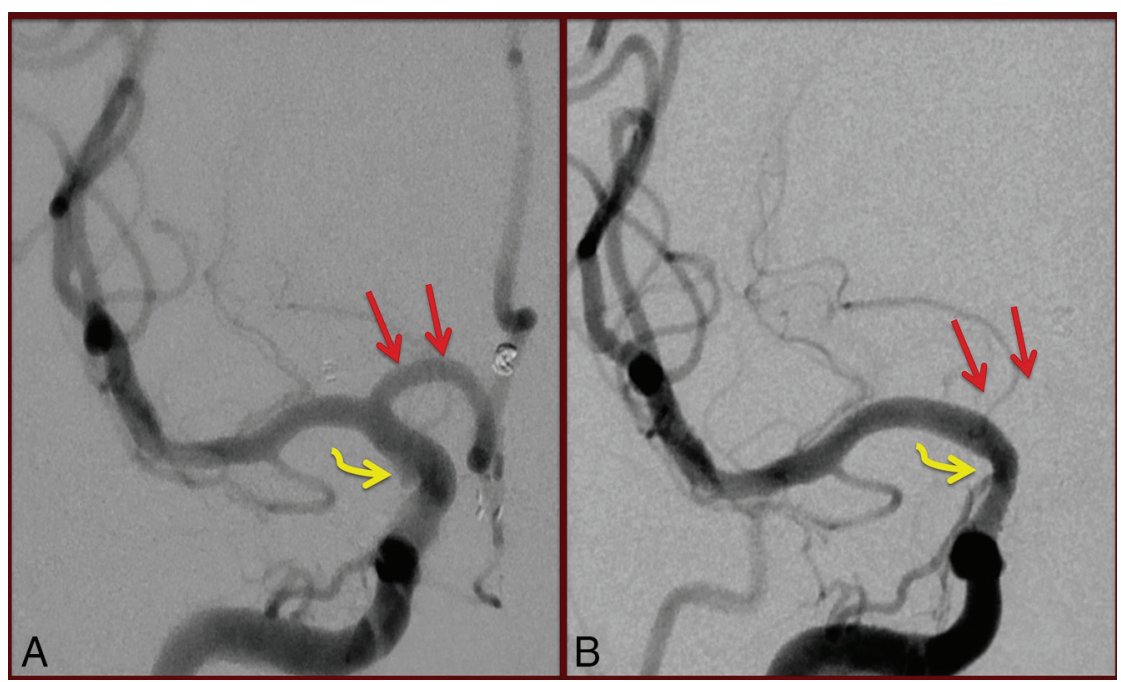

FIG 2. Anteroposterior magnified view $(A)$ of pre-PED right internal carotid artery angiography demonstrating gross patency of the $\mathrm{Al}$ segment of the right anterior cerebral artery (straight arrows). The target, irregular right anterior choroidal artery aneurysm is only partially visualized (curved arrow). On conventional angiography performed 6 months following PED deployment $(B)$, the $A 1$ segment of the right anterior cerebral artery no longer fills on right internal carotid artery injection. The target aneurysm is closed (curved arrow). The patient was asymptomatic, and the bilateral anterior cerebral arteries are noted to be filling via the left Al segment on left internal carotid artery injections (not shown). follow-up. Occluded branch vessels included 7 ophthalmic arteries $(5.8 \%)$ (Fig 1), 13 posterior communicating arteries (33.3\%), 4 Al anterior cerebral artery segments (36\%) (Fig 2), 2 anterior communicating arteries (66.6\%), 1 anterior choroidal artery (3.2\%), and $1 \mathrm{M} 2 \mathrm{MCA}$ arterial segment (100\%). Another 40 branch vessels (18.4\%) were significantly stenotic, demonstrating a reduction in both caliber and flow on conventional angiography. These vessels were frequently noted to demonstrate a severe focal stenosis at their origin and included an additional 28 ophthalmic arteries (22\%), 4 posterior communicating arteries (10\%), $4 \mathrm{~A} 1$ anterior cerebral arteries (36\%), and 2 posterior inferior cerebellar arteries (67\%). All branch vessel occlusions and stenoses in the cohort were asymptomatic.

Univariate analysis was performed to assess significant factors associated with branch vessel occlusion (Table 3). On initial review of the data, minimum, maximum, and average parent vessel size and PED diameter were all found to be significant predictors of branch occlusion in the entire cohort, as well as in the largest subset of ophthalmic arteries. We therefore elected to evaluate PED size to simplify the subsequent analysis and remove any covarying factors. By univariate analysis, variables associated with a higher rate of branch vessel occlusion include older age $(59.3 \pm 10.2$ versus $54.4 \pm 12.2$ years, $P=.04)$, smaller PED diameter $(4.0 \pm 0.5$ versus $3.5 \pm$ $0.5 \mathrm{~mm}, P<0.001$ ), and branch vessel types of the A1 anterior cerebral artery segment $(P=.021)$ and posterior communicating artery $(P<.001)$. Ophthalmic artery branch vessel type was associated with a significantly lower rate of occlusion than average $(P<.001)$. By multivariate modeling, only a smaller PED diameter $(P=.001)$ remained an independent predictor of branch vessel occlusion.

Receiver operating characteristic analysis was performed to demonstrate the diagnostic accuracy for parent vessel diameter to predict branch vessel occlusion in the entire cohort. Sensitivity and specificity values were calculated for a range of thresholds, with the resulting curve shown in Fig 3. The area under the 


\begin{tabular}{|c|c|c|c|c|c|}
\hline & Patent Branch Vessel & $\begin{array}{c}\text { Occluded Branch } \\
\text { Vessel }\end{array}$ & $\begin{array}{l}\text { Univariate } \\
P \text { Value }\end{array}$ & $\begin{array}{c}\text { Multivariate } \\
P \text { Value }\end{array}$ & OR $(95 \% \mathrm{Cl})$ \\
\hline Mean patient age (yr) & $54.4 \pm 12.2$ & $59.3 \pm 10.2$ & .04 & .179 & $1.03(0.99-1.07)$ \\
\hline Male & $26(13.8 \%)$ & $4(13.8 \%)$ & .996 & & \\
\hline Acutely ruptured target lesion & $7 / 188(3.7 \%)$ & $1 / 29(3 \%)$ & .942 & & \\
\hline Mean max aneurysm sac size & $5.6 \pm 3.8$ & $4.2 \pm 2.3$ & .067 & .117 & $0.87(0.73-1.04)$ \\
\hline Mean aneurysm neck size & $3.4 \pm 1.7$ & $3.0 \pm 1.2$ & .139 & & \\
\hline Mean procedural time (min) & $206 \pm 66$ & $214 \pm 60$ & .513 & & \\
\hline Mean fluoroscopy time (min) & $64 \pm 36$ & $62 \pm 27$ & .764 & & \\
\hline$>1$ PED deployed & $17 / 188(9.0 \%)$ & $1 / 29(3.4 \%)$ & .309 & & \\
\hline$\%$ PED classic & $107 / 125(86 \%)$ & $18 / 29(62.1 \%)$ & .601 & & \\
\hline Balloon angioplasty & $13 / 188(7 \%)$ & $1 / 29(7 \%)$ & .479 & & \\
\hline Mean PED diameter (mm) & $4.0 \pm 0.5$ & $3.5 \pm 0.5$ & $<0.001$ & .001 & $0.16(0.06-0.45)$ \\
\hline Mean $\Delta$ max size of parent vessel and PED & $0.22 \pm 0.19$ & $0.26 \pm 0.26$ & .405 & & \\
\hline Mean $\Delta$ min size of parent vessel and PED & $0.77 \pm 0.41$ & $0.67 \pm 0.40$ & .230 & & \\
\hline Branch vessel incorporation into aneurysm sac & $52 / 188(28 \%)$ & $9 / 29(31 \%)$ & .707 & & \\
\hline Mean periprocedural $\mathrm{P} 2 \mathrm{Y} 12$ & $127 \pm 68$ & $122 \pm 80$ & .379 & & \\
\hline Intraprocedural administration of abciximab & $17 / 188(9.0 \%)$ & $3 / 29(10.3 \%)$ & .821 & & \\
\hline Presence of endothelial hyperplasia & $43 / 185(23 \%)$ & $10 / 29(34 \%)$ & .192 & & \\
\hline Mean length of DSA follow-up (mo) & $18.3 \pm 9.9$ & $20.1 \pm 8.8$ & .351 & & \\
\hline $\mathrm{Al} A C A$ & $7 / 11(63.6 \%)$ & $4 / 11(36.3 \%)$ & .021 & .813 & $1.29(0.16-10.56)$ \\
\hline Anterior choroidal artery & $30 / 31(96.8 \%)$ & $1 / 31(3.2 \%)$ & .073 & .092 & $0.10(0.01-1.46)$ \\
\hline PcomA & $26 / 39(66.6 \%)$ & $13 / 39(33.3 \%)$ & $<.001$ & .205 & $3.26(0.52-20.28)$ \\
\hline Ophthalmic artery & $118 / 125$ (94\%) & $8 / 125(6 \%)$ & $<.001$ & 0.449 & $0.49(0.08-3.07)$ \\
\hline
\end{tabular}

Note:-ACA indicates anterior cerebral artery; max, maximum; min, minimum; PcomA, posterior communicating artery.

a Percentages reflect analysis per branch vessel.

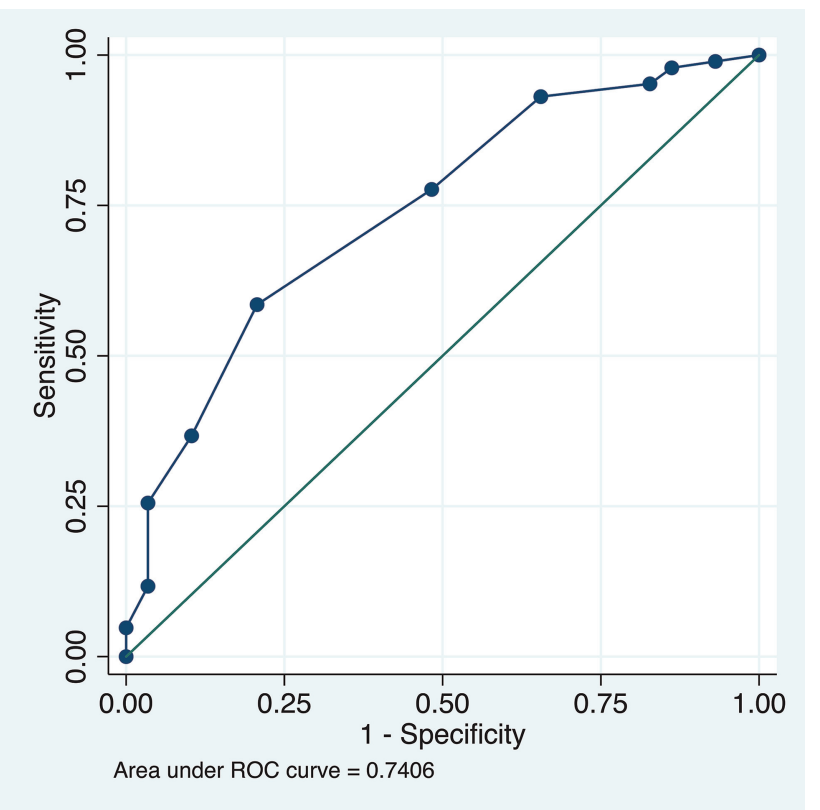

FIG 3. Receiver operating characteristic (ROC) curve demonstrating the diagnostic accuracy for parent vessel diameter to predict branch vessel occlusion in the entire cohort. The reference line is depicted as the solid line along the middle of the plot.

receiver operating characteristic curve was calculated (area under the curve $=0.741$ ), and a PED diameter of $4.0 \mathrm{~mm}$ was found to have the most favorable cut-point by the Youden method.

Because the factors associated with branch vessel occlusion may vary on the basis of the type of branch vessel in question, particularly the potential for collateral supply to the branch vessel territory, we elected to repeat our analysis for the largest subgroup of covered side branches, ophthalmic arteries $(n=125)$ (Table 4$)$. By univariate analysis, a smaller PED diameter $(4.1 \pm 0.5$ versus
$3.4 \pm 0.6 \mathrm{~mm}, P=.0006)$ and the use of intraprocedural abciximab (25\% versus $4.2 \%, P=.013$ ) were associated with ophthalmic artery occlusion. By multivariate analysis, a smaller PED diameter $(P<.001)$ again remained the only independent predictor of branch vessel occlusion.

Finally, because a significant minority of covered side branches demonstrated marked reductions in both caliber and flow on follow-up, we elected to repeat our analysis including these stenotic side branches with occluded vessels (Table 5 ). In univariate analysis, smaller PED diameter $(P=.013)$, intraprocedural use of abciximab for platelet aggregation $(P=.019)$, and A1 anterior cerebral artery segment branch vessel type $(P=.003)$ were significant predictors of covered side branch stenosis or occlusion. Anterior choroidal branch vessel type was associated with gross side branch patency $(P=<.001)$. On multivariate analysis, PED diameter $(P=.029)$ and the administration of intraprocedural abciximab $(P=.030)$ remained independent predictors of side branch stenosis or occlusion. Finally, anterior choroidal artery branch type $(P=.003)$ was an independent predictor of side branch patency.

\section{DISCUSSION}

To our knowledge, our study is the first to demonstrate that a smaller PED diameter is significantly associated with long-term covered side branch stenosis/occlusion following aneurysm treatment with a PED. Although prior authors have investigated the fate of covered side branches following PED deployment, none appear to have evaluated the impact of PED diameter. This correlation was present when we included only complete occlusion of covered side branches as well as when stenotic and occluded side branches were considered together. Furthermore, the association remained significant when analyzing the largest subgroup of side branches in our cohort, the ophthalmic arteries. The dependence 
Table 4: Factors impacting branch vessel patency_ophthalmic artery ${ }^{a}$

\begin{tabular}{|c|c|c|c|c|c|}
\hline & $\begin{array}{l}\text { Patent Branch } \\
\text { Vessel }\end{array}$ & $\begin{array}{c}\text { Occluded } \\
\text { Branch Vessel }\end{array}$ & $\begin{array}{c}\text { Univariate } \\
P \text { Value }\end{array}$ & $\begin{array}{c}\text { Multivariate } \\
P \text { Value }\end{array}$ & OR $(95 \% \mathrm{CI})$ \\
\hline Mean patient age (yr) & 53.8 & 60.1 & .119 & & \\
\hline Male & 15 (12.7\%) & $0(0 \%)$ & .283 & & \\
\hline Mean max aneurysm sac & $5.9 \pm 3.8$ & $5.8 \pm 3.3$ & .977 & & \\
\hline Mean aneurysm neck size & $3.6 \pm 1.6$ & $3.6 \pm 0.5$ & .998 & & \\
\hline Acutely ruptured target lesion & $4 / 118(3.7 \%)$ & $0 / 7(0 \%)$ & .597 & & \\
\hline Prior coiling & $29 / 118(24.6 \%)$ & $1 / 7(14.3 \%)$ & .438 & & \\
\hline Mean procedural time (min) & $200 \pm 63$ & $212 \pm 60$ & .628 & & \\
\hline Mean fluoroscopy time (min) & $63 \pm 37$ & $53 \pm 28$ & .429 & & \\
\hline$>1$ PED deployed & $10 / 118(8.5 \%)$ & $0 / 7(0 \%)$ & .391 & & \\
\hline$\%$ PED classic & $70 / 75(93.3 \%)$ & $5 / 75(6.7 \%)$ & .859 & & \\
\hline Balloon angioplasty & $9 / 118(7.6 \%)$ & $0 / 7(0 \%)$ & .418 & & \\
\hline Mean PED diameter (mm) & $4.1 \pm 0.5$ & $3.4 \pm 0.6$ & $<0.001$ & $<.001$ & $0.10(0.04-0.21)$ \\
\hline Mean $\Delta$ max size of parent vessel and PED & $0.23 \pm 0.18$ & $0.24 \pm 0.21$ & .887 & & \\
\hline Mean $\Delta$ min size of parent vessel and PED & $0.79 \pm 0.41$ & $0.79 \pm 0.48$ & .979 & & \\
\hline Branch vessel incorporation into aneurysm sac & $31 / 118(26.3 \%)$ & $2 / 7(29 \%)$ & .937 & & \\
\hline Mean periprocedural $\mathrm{P} 2 \mathrm{Y} 12$ & $136 \pm 68$ & $150 \pm 63$ & .597 & & \\
\hline Intraprocedural administration of abciximab & $5 / 118(4.2 \%)$ & $2 / 7(29 \%)$ & .013 & .194 & $4.09(0.49-34.19)$ \\
\hline Presence of endothelial hyperplasia & $26 / 115(22.6 \%)$ & $3 / 7(43 \%)$ & .337 & & \\
\hline Mean length of DSA follow-up (mo) & $17.9 \pm 9.9$ & $21.4 \pm 13.8$ & .682 & & \\
\hline
\end{tabular}

a Percentages reflect analysis per branch vessel.

Table 5: Factors impacting branch vessel patency vs occluded plus stenotic_entire cohort ${ }^{\text {a }}$

\begin{tabular}{|c|c|c|c|c|c|}
\hline & $\begin{array}{l}\text { Patent Branch } \\
\text { Vessel }\end{array}$ & $\begin{array}{c}\text { Occluded/Stenotic } \\
\text { Branch Vessel }\end{array}$ & $\begin{array}{l}\text { Univariate } \\
P \text { Value }\end{array}$ & $\begin{array}{c}\text { Multivariate } \\
P \text { Value }\end{array}$ & OR $(95 \% \mathrm{CI})$ \\
\hline Mean patient age (yr) & $54.6 \pm 12.6$ & $56.0 \pm 10.9$ & .426 & & \\
\hline Male & 19/148 (12.8\%) & $11 / 69(15.9 \%)$ & .537 & & \\
\hline Mean max aneurysm sac size & $6.0 \pm 4.0$ & $5.6 \pm 3.7$ & .096 & .415 & $0.94(0.82-1.09)$ \\
\hline Mean aneurysm neck size & $3.6 \pm 1.7$ & $3.4 \pm 1.2$ & .054 & .534 & $0.91(0.67-1.22)$ \\
\hline Acutely ruptured target lesion & $4 / 148(2.7 \%)$ & $4 / 69(5.8 \%)$ & .260 & & \\
\hline Mean procedural time (min) & $205 \pm 65$ & $210 \pm 66$ & .658 & & \\
\hline Mean fluoroscopy time (min) & $65 \pm 37$ & $60 \pm 30$ & .365 & & \\
\hline$>1$ PED deployed & $14 / 148(9.5 \%)$ & $4 / 69(5.8 \%)$ & .362 & & \\
\hline$\%$ PED classic & $60 / 148(40.5 \%)$ & $32 / 69(46.3 \%)$ & .418 & & \\
\hline Balloon angioplasty & $12 / 148(8.1 \%)$ & $2 / 69(2.9 \%)$ & .146 & & \\
\hline Mean PED diameter (mm) & $4.0 \pm 0.5$ & $3.8 \pm 0.5$ & .013 & .029 & $0.46(0.23-0.92)$ \\
\hline Mean $\Delta$ max size of parent vessel and PED & $0.21 \pm 0.17$ & $0.27 \pm 0.25$ & .057 & .142 & $3.60(0.65-19.89)$ \\
\hline Mean $\Delta$ min size of parent vessel and PED & $0.78 \pm 0.4$ & $0.70 \pm 0.42$ & .226 & & \\
\hline Branch vessel incorporation into aneurysm sac & $42 / 148(28.3 \%)$ & $16 / 69(27.5 \%)$ & .898 & & \\
\hline Mean periprocedural P2Y12 & $127 \pm 68$ & $125 \pm 74$ & .855 & & \\
\hline Intraprocedural administration of abciximab & $9 / 148(6.1 \%)$ & $11 / 69(15.9 \%)$ & .019 & .030 & $4.26(1.15-15.78)$ \\
\hline Presence of endothelial hyperplasia & $31 / 146(21.2 \%)$ & $22 / 68(32.4 \%)$ & .079 & .504 & $1.31(0.59-2.94)$ \\
\hline Mean length of DSA follow-up (mo) & $18.8 \pm 19.4$ & $18.0 \pm 8.2$ & .584 & & \\
\hline Al ACA & $3 / 11(27.2 \%)$ & $8 / 11(72.7 \%)$ & 0.003 & .114 & $3.12(0.76-12.81)$ \\
\hline Anterior choroidal artery & $30 / 31(96.8 \%)$ & $1 / 31(3.2 \%)$ & $<.001$ & .003 & $0.04(0.004-0.32)$ \\
\hline PcomA & $22 / 39(56.4 \%)$ & $17 / 39(43.6 \%)$ & .081 & .248 & $1.57(0.73-3.40)$ \\
\hline Ophthalmic artery & $90 / 125(71.2 \%)$ & $36 / 125(28.8 \%)$ & .23 & & \\
\hline
\end{tabular}

Note:-PcomA indicates posterior communicating artery; AcomA, anterior communicationg.

a Percentages reflect analysis per branch vessel.

of side branch patency on the PED diameter was not explained by a discrepancy in size between the PED and artery, the type of branch vessel covered, or the presence of endothelial hyperplasia in the flow-diverter construct on follow-up.

We believe that the association between PED diameter and delayed side branch occlusion or stenosis demonstrated in our cohort is best explained by the increased surface area metal coverage provided by smaller diameter devices. This increase in metal coverage is a natural consequence of all PEDs being constructed from the same number of metal strands of an approximately 30- $\mu \mathrm{m}$ diameter, which results in smaller devices having more metal density. Therefore, when appropriately sized, a smaller diameter PED will result in more metal coverage across the orifice of a jailed side branch compared with a larger device. ${ }^{15}$ Our results suggest that these differences in metal density between PEDs of varying caliber have a significant influence on the long-term patency of covered side arteries. The importance of metal coverage in determining long-term side branch patency is supported by findings of Rangel-Castilla et $\mathrm{al}^{12}$ of an association between the number of devices deployed and delayed side branch occlusion in a review of 127 anterior circulation branch vessels covered by PEDs. Although we did not find a similar association in our cohort, this likely reflects the small number of cases in our cohort using multiple PEDs.

However, the metal coverage provided by a deployed PED is dynamic, with the geometry of PED cells potentially influenced by 
multiple factors. These include the degree of oversizing of the device relative to the parent vessel, the course of the artery at the landing site (eg, linear versus curved), and finally the method of PED deployment (eg, aggressive pushing of the PED out of the microcatheter during delivery with resulting device compaction). ${ }^{15,16}$ Although these factors undoubtedly affected the metal surface area coverage provided by PEDs in our cohort, we believe that differences between individual cases were minimized by the use of a standard PED deployment technique in our group. For example, we match the PED diameter to that of the parent vessel as closely as possible because device oversizing (mild to moderate in degree) may impact treatment efficacy by increasing cell size and decreasing metal coverage across the aneurysm neck. ${ }^{15}$ Furthermore, we avoid excessive compaction or stretching of the PED during deployment because the former is not necessarily required to achieve subsequent aneurysm closure, while the latter may result in inadequate device apposition.

We also noted that intraprocedural administration of abciximab for acute platelet aggregation during PED deployment was associated with occluded or stenotic side branches on follow-up. These findings suggest a possible role for the degree of platelet inhibition in determining covered branch patency, though the immediate preprocedural P2Y12 value was not significantly associated with the status of branch vessels on follow-up.

Our results, when considered along with those of prior publications, suggest that the patency of covered side branches following PED treatment is determined by multiple factors, including hemodynamic factors, the amount of metal coverage at the jailed artery orifice, the degree of PED oversizing, and possibly the adequacy of platelet inhibition. A complex multifactorial process could explain, in part, why jailed side branch occlusion rates have varied so widely among prior publications $(3.5 \%-53.3 \%) .{ }^{10-13}$ However, of all these variables potentially impacting the fate of covered side branches, hemodynamic factors involving the jailed side branch are likely paramount. For example, in instances in which there is less potential for collateral flow to the covered side branch territory (eg, the anterior choroidal artery), previously reported long-term patency rates have been extremely high (approaching 100\%). ${ }^{13}$ Our own results corroborate these findings. However, in instances in which the availability of collateral flow to the side branch territory is more robust (eg, the ophthalmic artery or an A1 arterial segment), other factors, particularly the degree of metal coverage at the side branch orifice, also play an important role.

Consistent with the findings of prior authors, we noted that delayed side branch occlusion following PED deployment is most often clinically silent. This is presumably due to the rich collateral network available in many vascular territories in the brain, which is further served by the apparent slowly progressive nature of delayed side branch occlusions with the PED. However, symptomatic delayed side branch closures have been reported, and we have anecdotally noted a few cases in our practice (not included in the current study due to inadequate follow-up). ${ }^{11}$ Furthermore, some ischemic events following side branch occlusion may be missed because patients do not routinely undergo cross-sectional imaging or a detailed ophthalmologic examination following treatment. This idea is supported by Rouchaud et al, ${ }^{17}$ who found that ophthalmic complications were present in $39.3 \%$ of asymptomatic patients who underwent an extensive ophthalmologic examination in the first week following PED placement across the ophthalmic artery origin. Finally, the long-term consequences of branch vessel closures remain uncertain. For example, in patients with closure of an A1 segment of the anterior cerebral artery, there is the theoretic risk of inducing aneurysm growth in the region of the anterior communicating artery due to increased flow across the vessel from the contralateral A1 segment.

Due to the occasional symptomatic side branch occlusion, as well as the theoretic risks of silent ischemic events and longerterm sequelae, it is important for the neurointerventionalist to consider the potential for loss of covered side branches when evaluating a patient for possible aneurysm treatment with the PED. The risk of PED closure of covered side branches should be carefully weighed against the advantages of flow diversion, such as high aneurysm-occlusion rates, particularly when the lesion arises from a smaller caliber artery, which will necessitate the use of a smaller diameter, higher metal density device. In these instances, coil embolization or microsurgical repair, when feasible, may be preferable treatment options. Furthermore, in instances in which flow diversion is selected for aneurysm treatment, the potential for side branch occlusion should be discussed with the patient as part of the informed consent process.

The current study has several limitations, including its retrospective design and lack of data regarding patient-dependent variables such as smoking status and hypertension. However, prior reports have evaluated the impact of such variables on branch patency and did not find a significant association. The determination of branch artery stenosis was subjectively defined in our study and could include vessels moderately reduced in size to arteries that were nearly occluded. Furthermore, we did not evaluate the potential for collateral supply to a jailed side branch on a case-to-case basis (eg, evaluation of the contralateral A1 and anterior communicating artery caliber for all jailed A1 segments) because we are uncertain how to quantify the collateral potential in these instances. Instead, we believe that analysis by branch type is the best way to investigate the impact of collateral supply on long-term patency; therefore, we included branch type as a variable in our analysis. We also analyzed factors impacting branch patency in our largest subgroup of ophthalmic arteries and found results similar to those of the entire cohort. Finally, we did not evaluate other variables that may influence PED cell geometry/ metal coverage, including purposeful device compaction by the interventionalist or deployment along a vessel curve. However, we believe that the use of a uniform PED deployment technique in our group helped to minimize these factors. Future studies, possibly using flat panel CT interrogation of the deployed flow diverter, may help to further clarify whether there is a quantitative association between the percentage of metal coverage and delayed side branch stenosis/occlusion.

\section{CONCLUSIONS}

Smaller PED diameter is significantly associated with delayed side branch stenosis/occlusion following intracranial aneurysm treatment with the PED. The higher metal density of smaller PEDs compared with larger diameter devices best explains this associa- 
tion. Although hemodynamic factors, particularly the availability of collateral flow, are still paramount in determining the fate of covered side branches, the amount of metal coverage at the side branch orifice also plays an important role.

Disclosures: Timothy R. Miller—UNRELATED: Active/prior grants LVIS: Low-profile Visualized Intraluminal Support, a pivotal study of a MicroVention neurovascular self-expanding retrievable stent system in the treatment of wide-neck intracranial artery aneurysms, Sponsor: MicroVention; ULTRA: Ultra Coils from Start to Finish for the Endovascular Repair of Small Intracranial Aneurysms, Sponsor: Stryker; BARREL: prospective, multicenter, single-arm study of the Reverse Medical Barrel Vascular Reconstruction Device for adjunctive treatment to embolic coils for wide-neck, intracranial, bifurcating/branching aneurysms of the middle cerebral and basilar arteries, Sponsor: Medtronic; a prospective, multicenter, single-arm study to evaluate the safety and effectiveness of the Codman Enterprise Vascular Reconstruction Device and Delivery System when used in conjunction with endovascular coil embolization in the treatment of wide-neck saccular intracranial aneurysms; Sponsor: Medtronic; Apollo Onyx Delivery Micro Catheter Postmarket Safety Study; Sponsor: Medtronic; Trevo Retriever Registry: Post Market Surveillance Study; Sponsor: Stryker; Hydrogel Endovascular Aneurysm Treatment Trial (HEAT); Sponsor: Stryker; Gadobutrol Enhanced MRA of the Supra-Aortic Vessels, Sponsor: Bayer, Pipeline Flex versus classic retrospective study, Sponsor: Medtronic; Gaurav Jindal—UNRELATED: Grants/Grants Pending: Stryker Neurovascular and Medtronic, Comments: research grants. * Dheeraj Gandhi—UNRELATED: Royalties: Cambridge Press. *Money paid to the institution.

\section{REFERENCES}

1. Becske T, Kallmes DF, Saatci I, et al. Pipeline for uncoilable or failed aneurysms: results from a multicenter clinical trial. Radiology 2013; 267:858-68 CrossRef Medline

2. Chiu AH, Cheung AK, Wenderoth JD, et al. Long-term follow-up results following elective treatment of unruptured intracranial aneurysms with the Pipeline Embolization Device. AJNR Am J Neuroradiol 2015;36:1728-34 CrossRef Medline

3. Fischer S, Vajda Z, Aguilar Perez M, et al. Pipeline embolization device (PED) for neurovascular reconstruction: initial experience in the treatment of 101 intracranial aneurysms and dissections. Neuroradiology 2012;54:369-82 CrossRef Medline

4. Kallmes DF, Hanel R, Lopes D, et al. International retrospective study of the Pipeline embolization device: a multicenter aneurysm treatment study. AJNR Am J Neuroradiol 2015;36:108-15 CrossRef Medline

5. Saatci I, Yavuz K, Ozer C, et al. Treatment of intracranial aneurysms using the Pipeline flow-diverter embolization device: a single-cen- ter experience with long-term follow-up results. AJNR Am J Neuroradiol 2012;33:1436-46 CrossRef Medline

6. Albuquerque FC, Park MS, Abla AA, et al. A reappraisal of the Pipeline Embolization Device for the treatment of posterior circulation aneurysms. J Neurointervent Surg 2015;7:641-45 CrossRef Medline

7. Chalouhi N, Zanaty M, Whiting A, et al. Safety and efficacy of the Pipeline Embolization Device in 100 small intracranial aneurysms. J Neurosurg 2015;122:1498-502 CrossRef Medline

8. Pistocchi S, Blanc R, Bartolini B, et al. Flow diverters at and beyond the level of the circle of Willis for the treatment of intracranial aneurysms. Stroke 2012;43:1032-38 CrossRef Medline

9. Colby GP, Bender MT, Lin LM, et al. Endovascular flow diversion for treatment of anterior communicating artery region cerebral aneurysms: a single-center cohort of 50 cases. J Neurointervent Surg 2017;9:679-85 CrossRef Medline

10. Daou B, Valle-Giler EP, Chalouhi N, et al. Patency of the posterior communicating artery following treatment with the Pipeline Embolization Device. J Neurosurg 2017;126:564-69 CrossRef Medline

11. Chalouhi N, Daou B, Kung D, et al. Fate of the ophthalmic artery after treatment with the Pipeline Embolization Device. Neurosurgery 2015;77:581-84; discussion 584 CrossRef Medline

12. Rangel-Castilla L, Munich SA, Jaleel N, et al. Patency of anterior circulation branch vessels after Pipeline embolization: longer-term results from 82 aneurysm cases. J Neurosurg 2017;126:1064-69 CrossRef Medline

13. Raz E, Shapiro M, Becske T, et al. Anterior choroidal artery patency and clinical follow-up after coverage with the Pipeline Embolization Device. AJNR Am J Neuroradiol 2015;36:937-42 CrossRef Medline

14. Bhogal P, Ganslandt $\mathrm{O}$, Bäzner $\mathrm{H}$, et al. The fate of side branches covered by flow diverters: results from 140 patients. World Neurosurg 2017;103:789-98 CrossRef Medline

15. Shapiro M, Raz E, Becske T, et al. Variable porosity of the Pipeline embolization device in straight and curved vessels: a guide for optimal deployment strategy. AJNR Am J Neuroradiol 2014;35:727-33 CrossRef Medline

16. Ma D, Xiang J, Choi $\mathrm{H}$, et al. Enhanced aneurysmal flow diversion using a dynamic push-pull technique: an experimental and modeling study. AJNR Am J Neuroradiol 2014;35:1779-85 CrossRef Medline

17. Rouchaud A, Leclerc O, Benayoun Y, et al. Visual outcomes with flow-diverter stents covering the ophthalmic artery for treatment of internal carotid artery aneurysms. AJNR Am J Neuroradiol 2015; 36:330-36 CrossRef Medline 